\title{
Quantum Optimal Control of Bose-Einstein-Condensates in 3D
}

This paper was downloaded from TechRxiv (https://www.techrxiv.org).

\section{LICENSE}

CC BY-NC-SA 4.0

SUBMISSION DATE / POSTED DATE

25-04-2021 / 29-04-2021

\section{CITATION}

Wang, Quan-Fang (2021): Quantum Optimal Control of Bose-Einstein-Condensates in 3D. TechRxiv. Preprint. https://doi.org/10.36227/techrxiv.14480475.v1

$\mathrm{DOI}$ 


\title{
Quantum Optimal Control of Bose-Einstein-Condendates in $3 D^{\dagger}$
}

\author{
Quan-Fang Wang*
}

\begin{abstract}
Quantum control of Bose-Einstein-Condensates (BEC) had been found for one and two dimension cases. Firstly in this paper, we want to control BEC at electro-magnetic field in three dimension optical lattice theoretically. The trapped particles in constructed lattices can be made and controlled by optical pulse, and known as molasses. It is evident that quantum control theory is worked for physical particles in BEC status. It is a survey with system control and quantum particle physics. Future work is to focus on collaborating with real laboratory. Keywords. Quantum control, Bose-Einstein-Condensates, Optical lattices, Molasses.
\end{abstract}

\section{INTRODUCTION}

One and two dimension quantum control theory of BoseEinstein-Condensates give us the confidence to do the investigation on the three dimension, 3D. First of all, observing the contributed works on BEC, the existing results are concentrated on the physical field, and there is few ones on the quantum control for condensates. Actually, the status of particles condensate is well known and sufficiently considered in the research of physical/chemical areas, and seldom to be taken into account in system control as well as applied science. As a physical experiment, whether our study is sufficient for BEC? Can we control particles of BEC as it taking place in lab? In fact, controlling is being in the entire experiment process as we using optical lattice to drive a particle status from its ground state to a critical state: condensates. As we compose these optical lattices in lab setting, and shape the laser pulses, the proper directions and appropriate angles of optical pulse can be selected for making a successful molasses in practical condensates. All the factors would be regarded as controllers, but, particularly, the optical lattice and shaped laser pulse are the exact control inputs what we mean in quantum system control. Second is to abstract and extract these matters as mathematical physicsmodel, and to do optimal control approach for BEC. Vice versa, final is to utilize theoretic conclusion as guidance to further real experiments as well as prediction to unknown physical experiments what we want to do next.

Originally, in this paper, we intend to control of BoseEinstein-Condensates for particles in three dimension optical lattices at electro-magnetic field . Fortunately, a great deal literatures and contributed papers can be cited as our references, especially, the articles in physical field are the most benefit of our work. According its appeared order, one can find detailed papers in here. (cf. [3], [7], [8])

\footnotetext{
${ }^{\dagger}$ Chinese University of Hong Kong, Shatin, N. T. Hong Kong.

*E-mail: quanfangwang@hotmail.com
}

Several sections are in this paper. Section II is to deal with the Bose-Einstein-Condensates at the physics field. Section III is to do mathematical setting for BEC in Hilbert space. Section IV is quantum control for BEC using variational method. Section $\mathrm{V}$ is conclusion of our work.

\section{BOSE-EINSTEIN CONDENSATES}

As an ultra-cold vapor of bosonic atoms are trapped in electro-magnetic well, one can create pure condensates as they are cooling to the temperature than a BEC criticalness. As that taking place, a BEC is located into a optical lattice potential, which could be setting up experimentally by a fardetuned laser pulse. Optical lattices is known as molasses surrounding the particles. Indeed, quantum system for ultracold atoms is unique in precision and flexibility for experimental controlling.

Suppose $\mathbf{n}$ number particles are in optical lattices, each particle as $P_{n}$ for $n=1,2, \ldots, \mathbf{n}$. Set $\mathbf{x}=\left(x_{1}, x_{2}, x_{3}\right)$ in $\mathbf{R}^{3}$. Bose-Einstein-Condensates usually can be taken as the Gross Pitaevskii (GP) equation, a cubically nonlinear Schrödinger equation (cf. [6])

$$
\begin{aligned}
& \mathbf{i} \hbar \psi_{t}^{n} \\
= & -\frac{\hbar^{2}}{2 M} \Delta \psi^{n}+v_{1}^{n}(\mathbf{x}) \psi^{n}+v_{2}^{n}(\mathbf{x}) \psi^{n}+\mathbf{n} v\left|\psi^{n}\right|^{2} \psi^{n},
\end{aligned}
$$

$n=1,2, \ldots, \mathbf{n}$, where $\hbar$ is the Planck constant, $M$ is the atomic mass, $\psi^{n}(t, \mathbf{x})$ is the condensate wave function of one particle $P_{n}$ in BEC, $\mathbf{n}$ is the number of atoms in the condensate, and

$$
v=4 \pi \hbar^{2} a / M,
$$

where $a \in \mathbf{R}$ is the characteristic scatter length. The external potential $v_{1}^{n}(\mathbf{x})$ is the electro-magnetic trap needed for the experimental realization of a particle $P_{n}$ in BEC. In general,

$$
v_{1}^{n}(\mathbf{x})=M \omega_{0}^{2} \frac{|\mathbf{x}|^{2}}{2}
$$

for $\omega_{0} \in \mathbf{R}$. In GP equation (1), a potentials for particle $P_{n}$ used in physical experiments is then given by [6] as

$$
v_{2}^{n}(\mathbf{x})=p \sum_{\tau=1}^{3} \frac{\hbar^{2} \mathbf{x}^{2}}{M} \sin ^{2}\left(x_{\tau} x_{\tau}\right), x_{\tau} \in \mathbf{R},
$$

where $\mathbf{x}=\left(x_{1}, x_{2}, x_{3}\right)$ is the wave vector of the applied laser field, and $p>0$ is a dimensionless parameter for the expressing the depth of optical lattice. 


\section{MATHEMATICAL SETTING}

Quantum control for BEC as Gross-Pitaevskii equation (1) is to do mathematically setting to our problem into general Hilbert spaces.

At 3 dimensions space, $\Omega$ be an open bounded set of $\mathbf{R}^{3}$ and $Q=(0, T) \times \Omega$ for $T>0$. That is $(\mathbf{x}, t) \in Q$. In (1), $v_{1}^{n}(\mathbf{x})$ and $v_{2}^{n}(\mathbf{x})$ are the control inputs of particle $P_{n}$, and set $\mathbf{v}^{n}=\left(v_{1}^{n}, v_{2}^{n}\right)$ if necessary. Clearly, $\mathbf{v}^{n}(\mathbf{x})$ is depended on spatial variable $\mathbf{x}$, and independent of time $t$. Introduce two Hilbert spaces

$$
H=L^{2}(\Omega), V=H_{0}^{1}(\Omega)
$$

equipped with usual norms and inner products (cf. [4], [17]). Thus, the embeddings in Gelfand triple spaces

$$
V \hookrightarrow H \hookrightarrow V^{\prime}
$$

are continuous, dense and compact.

Actually, the solution $\psi^{n}$ of BEC system (1) is a complex function belonging to a complex function space $V$ mathematically, we disregard the difference in here for losting of generality to a real BEC status. $V$ is representing real, complex spaces associate with real, complex norms and inner products, respectively. Precisely, one can do optimal control for real part of solution $\psi^{n}$ in numerical approach.

Suppose $\mathcal{V}=L^{2}(\Omega)$ is the space of laser controls $v_{1}^{n}$ and $v_{2}^{n}, \mathcal{V}_{a d}$ is a closed and convex admissible set of $\mathcal{V}$. Assume initial state of particle $P_{n}$ as $\psi^{n}\left(v_{1}^{n}, v_{2}^{n}, 0\right)=\psi_{0}^{n}$. Cost function of a particle $P_{n}$, associated with GP system (1) is given by

$$
\begin{aligned}
J\left(v_{1}^{n}, v_{2}^{n}\right)= & \frac{\epsilon_{1}}{2} \int_{\Omega} \psi_{f}^{n}\left(v_{1}^{n}, v_{2}^{n}\right) \psi_{\text {target }}^{n}\left(v_{1}^{n}, v_{2}^{n}\right) d \mathbf{x} \\
& +\epsilon_{2} \int_{\mathcal{V}}\left(v_{1}^{n}\right)^{2} d \mathbf{x}+\epsilon_{3} \int_{\mathcal{V}}\left(v_{2}^{n}\right)^{2} d \mathbf{x},
\end{aligned}
$$

where $v_{1}^{n}, v_{2}^{n} \in \mathcal{V}_{a d}, \psi_{\text {target }}^{n}\left(v_{1}^{n}, v_{2}^{n}\right)$ is target state, $\psi_{f}^{n}\left(v_{1}^{n}, v_{2}^{n}\right)$ is observed final state of particle $P_{n}$ at final time $t_{f} . \epsilon_{i}, i=1,2,3$ are weighted coefficients for balancing the values of system and running costs, a kind of Tihonov regularity for control theory.

Quantum optimal control is to:

i). find quantum optimal control $v_{1}^{n *}$ or $v_{2}^{n *}, n=1,2, \ldots, \mathbf{n}$ for GP system (1).

ii). find optimality system for $v_{1}^{n *}, v_{2}^{n *}$.

For particle $P_{n}$, the $v_{1}^{n *}$ and $v_{2}^{n *}$ are called quantum optimal control for system (1) subject to cost function (2), set $\mathbf{v}^{*}=$ $\left(v_{1}^{n *}, v_{2}^{n *}\right)$ if needed.

Cite [2], [15] to give two definitions.

Definition 1: For all $n=1,2, \ldots, \mathbf{n}$, define the solution space by

$$
\begin{aligned}
& W\left(0, T ; V, V^{\prime}\right) \\
= & \left\{\psi^{n} \mid \psi^{n} \in L^{2}(0, T ; V), \psi^{n^{\prime}} \in L^{2}\left(0, T ; V^{\prime}\right)\right\} .
\end{aligned}
$$

For $\psi^{n}, \phi^{n} \in W\left(0, T ; V, V^{\prime}\right)$ and $n=1,2, \ldots, \mathbf{n}$, its norm and inner product can be defined as:

$$
\begin{aligned}
& \left\|\psi^{n}\right\|_{W\left(0, T ; V, V^{\prime}\right)}^{2} \\
= & \left\|\psi^{n}\right\|_{L^{2}\left(0, T ; V, V^{\prime}\right)}^{2}+\left\|\psi^{n \prime}\right\|_{L^{2}\left(0, T ; V^{\prime}\right)}^{2}, \\
& \left(\psi^{n}, \phi^{n}\right)_{W\left(0, T ; V, V^{\prime}\right)} \\
= & \left(\psi^{n}, \phi^{n}\right)_{L^{2}(0, T ; V)}+\left(\psi^{n \prime}, \phi^{n \prime}\right)_{L^{2}\left(0, T ; V^{\prime}\right)} .
\end{aligned}
$$

$W\left(0, T ; V, V^{\prime}\right)$ is a Hilbert space.

Definition 2: A function $\psi^{n}$ is called weak solution of (1) for particle $P_{n}$ and $n=1,2, \ldots, \mathbf{n}$, if $\psi^{n} \in W\left(0, T ; V, V^{\prime}\right)$ and satisfy

$$
\begin{aligned}
& \int_{0}^{T} \int_{\Omega} \mathbf{i} \hbar \psi_{t}^{n} \phi^{n} d \mathbf{x} d t \\
= & \frac{\hbar^{2}}{2 M} \int_{0}^{T} \int_{\Omega} \psi_{\mathbf{x}}^{n} \phi_{\mathbf{x}}^{n} d \mathbf{x} d t+\int_{0}^{T} \int_{\Omega} v_{1}^{n}(\mathbf{x}) \psi^{n} \phi^{n} d \mathbf{x} d t \\
+ & \int_{0}^{T} \int_{\Omega} v_{2}^{n}(\mathbf{x}) \psi^{n} \phi^{n} d \mathbf{x} d t+\mathbf{n} v \int_{0}^{T} \int_{\Omega}\left|\psi^{n}\right|^{2} \psi^{n} \phi^{n} d \mathbf{x} d t,(3)
\end{aligned}
$$

where $\phi^{n}$ is a arbitrary test function by the means of distribution in space $\mathcal{D}^{\prime}(0, T)$,

$$
\phi^{n} \in C^{1}(0, T ; V), \phi^{n}(T)=0 .
$$

\section{QUANTUM CONTROL FOR BEC}

Quantum control for Bose-Einstein-Condensates and numerical approach had already been taken account into papers $[11,12,13,14,15]$. Our task in this article is to fully in proof of quantum optimal control for BEC theoretically.

Refer [4], as in former paper [15], from weak form (3) to get the Theorems.

Theorem 3: For given initial state $\psi_{0}^{n} \in V$ of particle $P_{n}$ and $n=1,2, \ldots, \mathbf{n}$, there exists weak solution $\psi^{n} \in$ $W\left(0, T ; V, V^{\prime}\right)$ for GP system (1), and satisfy weak form (3).

Proof. Faedo-Galerkin method is used as in [4].

Step 1. Construct an approximate solution for the system (1). Since $V \hookrightarrow H$ is compact, then there exists an orthogonal basis of $H,\left\{w_{j}\right\}_{j=1}^{\infty}$ consisting of eigenfunctions of $A=\Delta$, such that $A w_{j}=\lambda_{j} w_{j}$ for all $j$,

$$
0<\lambda_{1} \leq \lambda_{2} \leq \cdots, \lambda_{j} \rightarrow \infty
$$

as $j \rightarrow \infty . G_{i}$ is the orthogonal projection of $H$ (or $V$ ) onto the space spanned by $\left\{w_{1}, \cdots, w_{i}\right\}$. For each $i \in N$, an approximate solution is defined for (1) by

$$
\psi_{i}^{n}(t)=\sum_{j=1}^{i} a_{i j}^{n}(t) w_{j}, \quad n=1,2, \ldots, \mathbf{n}
$$

where $a_{i j}^{n}(t)$ is real value coefficient function. Taking $\phi^{n}=$ $w_{j}$ in weak form (3), then approximate solution $\psi_{i}^{n}(t)$ satisfy 
the ordinary differential equation $(1 \leq i \leq N)$ given by

$$
\left\{\begin{array}{l}
\int_{0}^{T} \int_{\Omega} \mathbf{i} \hbar \psi_{i, t}^{n} w_{j} d \mathbf{x} d t \\
=\frac{\hbar^{2}}{2 M} \int_{0}^{T} \int_{\Omega} \nabla \psi_{i}^{n} \nabla w_{j} d \mathbf{x} d t+\int_{0}^{T} \int_{\Omega} v_{1}^{n}(\mathbf{x}) \psi_{i}^{n} w_{j} d \mathbf{x} d t \\
+\int_{0}^{T} \int_{\Omega}^{T} v_{2}^{n}(\mathbf{x}) \psi_{i}^{n} w_{j} d \mathbf{x} d t+\mathbf{n} v \int_{0}^{T} \int_{\Omega}\left|\psi_{i}^{n}\right|^{2} \psi_{i}^{n} w_{j} d \mathbf{x} d t \\
\psi_{i}^{n}(0)=\psi_{i 0}^{n},
\end{array}\right.
$$

Standard theory of ODE ensure that system (4) had unique local solution $\left\{\psi_{i}^{n}\right\}$ for $i=1,2, \cdots, N$.

Step 2. For given $\psi_{i 0}^{n}$, there exists a $\psi_{0}^{n} \in V$ such that

$$
\psi_{i 0}^{n} \rightarrow \psi_{0}^{n} \quad \text { strongly in } V \text {. }
$$

A unique function $\psi^{n}$ exists in $V$, same argument to $\left\{\psi_{i}^{n}\right\}$ as $\left\|\psi_{i}^{n}-\psi^{n}\right\|_{V} \rightarrow 0$, and

$$
\psi_{i}^{n} \text { is bounded in } L^{\infty}(0, T ; V) .
$$

Step 3. Suppose that $\left\{\psi_{i}^{n}\right\}$ and $\left\{\psi_{k}^{n}\right\}$ are two solutions of (1) with initial function $\left\{\psi_{0}^{n}\right\}$. Citing (3), one can find that

$$
\begin{aligned}
& \frac{\mathbf{i} \hbar}{2} \frac{d}{d t} I^{i k}(t)+\frac{\hbar^{2}}{2 M}\left\|\psi_{i}^{n}-\psi_{k}^{n}\right\|_{V}^{2} \\
\leq & C(t)\left(\left\|\psi_{i}^{n}-\psi_{k}^{n}\right\|^{2}+\mathbf{n} v\left\|\left|\psi_{i}^{n}\right|^{2} \psi_{i}^{n}-\left|\psi_{k}^{n}\right|^{2} \psi_{k}^{n}\right\|^{2}\right)
\end{aligned}
$$

Set

$$
C(t)=\max \left\{\left(v_{1}^{n}(t)+v_{2}^{n}(t)\right), 2 \mathbf{n} v\left(\|\left.\psi_{i}^{n}\right|^{2}+\left|\psi_{k}^{n}\right|^{2}\right)\right\},
$$

and

$$
I^{i k}(t)=\left\|\psi_{i}^{n}(t)-\psi_{k}^{n}(t)\right\|^{2}+\left\|\psi_{i}^{n}(t)-\psi_{k}^{n}(t)\right\|_{V}^{2} .
$$

By the positivity of second term of (5) to get that

$$
\frac{d}{d t} I^{i k}(t) \leq C^{\prime}(t) I^{i k}(t)
$$

Set $\lambda=\max \left\{\frac{\hbar}{2}, \frac{\hbar^{2}}{2 M}\right\}$ and $C^{\prime}(t)=C(t) / \lambda$. GronwallBellman inequality to yield that:

$$
I^{i k}(t) \leq \exp \left(\int_{0}^{T} C^{\prime}(t) d t\right) I^{i k}(0) .
$$

From (4) and (6) to deduce that

$$
\psi_{i}^{n} \rightarrow \psi^{n} \quad \text { in } \quad C\left(0, T ; L^{2}(\Omega)\right) .
$$

Finally, $C\left(0, T ; L^{2}(\Omega)\right) \subset W\left(0, T ; V, V^{\prime}\right)$ can assure the proof of Theorem 3 .

Theorem 4: For given $\psi_{0}^{n} \in V$ of particle $P_{n}$ and $n=$ $1,2, \ldots, \mathbf{n}$, there exists at least one quantum optimal control pair $\mathbf{v}^{n *}=\left(v_{1}^{n *}, v_{2}^{n *}\right)$ for GP system (1) subject to cost function (2).

Proof. Set $J=\inf _{\mathbf{v}^{n} \in \mathcal{V}_{a d}} J\left(\mathbf{v}^{n}\right)$, since $\mathcal{V}_{a d}$ is non-empty, there is a sequence $\left\{\mathbf{v}_{k}^{n}\right\}=\left\{\left(v_{1 k}^{n}, \mathbf{v}_{2 k}^{n}\right)\right\}$ in $\mathcal{V}_{a d}^{n} \times \mathcal{V}_{a d}^{n}$ such that

$$
\inf _{\mathbf{v}^{n} \in \mathcal{V}_{a d}^{n} \times \mathcal{V}_{a d}^{n}} J\left(\mathbf{v}^{n}\right)=\lim _{k \rightarrow \infty} J\left(\mathbf{v}_{k}^{n}\right)=J .
$$

Clearly, $\left\{J\left(\mathbf{v}^{n}\right)\right\}$ is bounded in $\mathbf{R}^{+}$. Since $\mathcal{V}_{a d}$ is bounded closed and convex, from (7), there exist a subsequences of $\left\{\mathbf{v}_{k}^{n}\right\}, \mathbf{v}_{k}^{n}=\left(v_{1 k}^{n}, v_{2 k}^{n}\right)$ (rewritten as itself) can be extracted, and $\mathbf{v}^{n *}=\left(v_{1}^{n *}, v_{2}^{n *}\right) \in \mathcal{V}_{a d}^{n} \times \mathcal{V}_{a d}^{n}$ such that

$$
\mathbf{v}_{k}^{n} \rightarrow \mathbf{v}^{n *} \text { weakly in } \mathcal{V} \text { as } k \rightarrow \infty \text {. }
$$

For $\psi^{n}$ and $\mathbf{v}^{n}=\left(v_{1}^{n}, v_{2}^{n}\right)$, the boundedness of $\mathcal{V}_{a d}$ to get that

$$
\psi^{n}\left(\mathbf{v}_{k}^{n}\right) \text { is bounded in } W\left(0, T ; V, V^{\prime}\right) .
$$

Setting $\psi^{n *}=\psi^{n}\left(\mathbf{v}^{n *}\right)$, there exist a subsequence (rewritten as itself) of $\psi^{n}\left(\mathbf{v}_{k}^{n}\right)$ can be extracted, and $\psi^{n *} \in$ $W\left(0, T ; V, V^{\prime}\right)$ such that

$$
\psi^{n}\left(\mathbf{v}_{k}\right) \rightarrow \psi^{n *} \quad \text { weakly in } W\left(0, T ; V, V^{\prime}\right)
$$

as $k \rightarrow \infty$. Since the embedding $V \hookrightarrow H$ is compact, there is $\bar{\psi}^{n}$ in $H$, from (9) and Aubin-Lions-Temam compactness embedding theorem (cf. [10]) to deduce that

$$
\psi^{n}\left(\mathbf{v}_{k}\right) \rightarrow \bar{\psi}^{n} \quad \text { strongly in } L^{2}(0, T ; H)
$$

as $k \rightarrow \infty$, and get the convergences for $\psi^{n}$

$$
\begin{array}{ll}
\psi_{t}^{n}\left(\mathbf{v}_{k}\right) \rightarrow \bar{\psi}_{t}^{n} & \text { weakly in } L^{2}\left(0, T ; V^{\prime}\right), \\
\nabla \psi^{n}\left(\mathbf{v}_{k}\right) \rightarrow \nabla \bar{\psi}^{n *} & \text { weakly in } L^{2}(0, T ; H),
\end{array}
$$

as $k \rightarrow \infty$. Set $\psi_{k}^{n}=\psi^{n}\left(\mathbf{v}_{k}\right)$, therefore, by taking test function $\phi^{n}$ in the definition of weak solution (3) to find

$$
\left\{\begin{array}{l}
\int_{0}^{T} \int_{\Omega}\left[-\mathbf{i} \hbar \sum_{i=1}^{N} \psi_{k}^{n} \frac{\partial \phi^{n}}{\partial t}\right. \\
-\frac{\hbar^{2}}{2 M} \sum_{i=1}^{N} \frac{\partial \psi_{k}^{n}}{\partial \mathbf{x}} \frac{\partial \phi^{n}}{\partial \mathbf{x}}+\sum_{i=1}^{N}\left(v_{1}(t)+v_{2}(t)\right) \psi_{k}^{n} \phi^{i} \\
\left.+\mathbf{n} v \sum_{i=1}^{N}\left|\psi_{k}^{n}\right|^{2} \psi_{k}^{n} \phi^{i}\right] d \mathbf{x} d t
\end{array}\right.
$$

If using (8), we take $k \rightarrow \infty$ in (11) to yield that

$$
\left\{\begin{array}{l}
\int_{0}^{T} \int_{\Omega}\left[-\mathbf{i} \hbar \sum_{i=1}^{N} \bar{\psi}^{n} \frac{\partial \phi^{n}}{\partial t}\right. \\
-\frac{\hbar^{2}}{2 M} \sum_{i=1}^{N} \frac{\partial \bar{\psi}^{n}}{\partial \mathbf{x}} \frac{\partial \phi^{n}}{\partial \mathbf{x}}+\sum_{i=1}^{N}\left(v_{1}(t)+v_{2}(t)\right) \bar{\psi}^{n} \phi^{n} \\
\left.+\mathbf{n} v \sum_{i=1}^{N}\left|\bar{\psi}^{n}\right|^{2} \bar{\psi}^{n} \phi^{n}\right] d \mathbf{x} d t
\end{array}\right.
$$

for all $\phi^{n} \in C^{1}(0, T ; V)$.

That is, the limit $\bar{\psi}^{n}$ satisfy (12) for all test functions $\phi^{n} \in V$ in the sense of $\mathcal{D}^{\prime}(0, T)$, which is distribution on $(0, T)$. From the uniqueness of weak solution for system (1) to attain

$$
\bar{\psi}^{n}=\psi^{n}\left(\mathbf{v}^{*}\right) .
$$

From convergence (10) to get that

$$
\begin{array}{ll}
\psi^{n}\left(\mathbf{v}_{k}\right), \rightarrow \psi^{n}\left(\mathbf{v}^{*}\right) & \text { strongly in } L^{2}(Q), \\
\psi_{f}^{n}\left(\mathbf{v}_{k}\right) \rightarrow \psi_{f}^{n}\left(\mathbf{v}^{*}\right) & \text { strongly in } L^{2}(\Omega)
\end{array}
$$

as $k \rightarrow \infty$. Since the norm $\|\cdot\|_{L 2(\Omega)}$ are lower semicontinuous in weak topology of $L^{2}(\Omega)$. Via (13) and (14) to find that

$$
\liminf _{k \rightarrow \infty}\left\|\psi_{f}^{n}\left(\mathbf{v}_{k}\right)-\psi_{\text {target }}^{n}\right\|_{V}^{2} \geq\left\|\psi_{f}^{n}\left(\mathbf{v}^{*}\right)-\psi_{\text {target }}^{n}\right\|_{V}^{2},
$$


In constrast, from the weak convergence (8) to know

$$
\liminf _{k \rightarrow \infty}\left(\mathbf{v}_{k}, \mathbf{v}_{k}\right) \mathcal{v} \times \mathcal{V} \geq\left(\mathbf{v}^{*}, \mathbf{v}^{*}\right) \mathcal{V} \times \mathcal{V}
$$

Since $J$ is weakly lower semi-continous, we obtain

$$
J=\liminf _{k \rightarrow \infty} J\left(\mathbf{v}_{k}\right) \geq J\left(\mathbf{v}^{*}\right),
$$

and hence $J\left(\mathbf{v}^{*}\right)=\inf _{\mathbf{v} \in \mathcal{V}_{a d}} J(\mathbf{v})$. That is, $\mathbf{v}^{*}=\left(v_{1}^{*}, v_{2}^{*}\right)$ is quantum optimal control subject to criteria function (2). It is the proof of Theorem 4.

Theorem 5: For given initial state $\psi_{0}^{n} \in V$, control problem for GP system (1) associated with cost function (2), then the quantum optimal control $\mathbf{v}^{n *}$ is characterized by simultaneously optimality system as

$$
\begin{aligned}
& \left\{\begin{array}{l}
\mathbf{i} \hbar \psi_{t}^{n}=-\frac{\hbar^{2}}{2 M} \Delta \psi^{n}+\mathbf{v}^{n *}(\mathbf{x}) \psi^{n}+\mathbf{n} v\left|\psi^{n}\right|^{2} \psi^{n} \text { in } \mathrm{Q}, \\
\psi^{n}\left(\mathbf{v}^{n *}, 0\right)=\psi_{0}^{n} \text { on } \Omega,
\end{array}\right. \\
& \left\{\begin{array}{l}
\mathbf{i} \hbar p_{t}^{n}=-\frac{\hbar^{2}}{2 M} \Delta p^{n}+2 \mathbf{n} v\left|\psi^{n}\right| \psi^{n} p^{n}+\mathbf{n} v\left|\psi^{n}\right|^{2} p^{n} \text { in } \mathrm{Q}, \\
\mathbf{i} p_{f}^{n}=\psi_{f}^{n}\left(\mathbf{v}^{n *}\right)-\psi_{\text {target }}^{n} \text { on } \Omega
\end{array}\right. \\
& \int_{Q} p^{n}\left(\mathbf{v}^{n *}\right)\left(\mathbf{v}-\mathbf{v}^{n *}\right) d \mathbf{x} d t+\left(\mathbf{v}^{n *}, \mathbf{v}^{n}-\mathbf{v}^{n *}\right) \mathcal{V} \geq 0
\end{aligned}
$$

for all $\mathbf{v}^{n}=\left(v_{1}^{n}, v_{2}^{n}\right) \in \mathcal{V}_{a d}^{2}$. In here, $p^{n} \in W\left(0, T ; V, V^{\prime}\right)$ is solution of the adjoint system (16) corresponding to $\psi^{n}$ in GP system (15). $p_{f}^{n}$ is the terminal state of adjoint solution $p^{n}$ at final time $t_{f}$.

It is well known that the inequality (17) is necessary optimality condition for $\mathbf{v}^{n *}=\left(v_{1}^{n *}, v_{2}^{n *}\right)$ of particle $P_{n}$ and $n=1,2, \ldots, \mathbf{n}$.

Proof is using the adjoint system for substituting in general necessary optimality condition $J^{\prime}\left(\mathbf{v}^{*}\right)\left(\mathbf{v}-\mathbf{v}^{*}\right) \geq 0$. Omit details in here.

For numerical experiments, updated conjugate gradient method and finite element approach is finding quantum optimal control $\mathbf{v}^{n *}$. Article [15] is for 2D case.

\section{CONCLUSIONS}

Quantum control for Bose-Einstein-Condensate is seeking optimal solution in 3D optical lattices at electro-magnetic field. Currently, it is demanded for a complementary of computational approach and real laboratory experiments to evidence our theory (cf. [12], [13], [14]) and books [16], [17].

Future research is opening for real condensates in physical/chemical fields. The present contribution can be found as [1], [5], [9], [11]. Can our theoretical controlling play a role in computational approach and in laboratory experiments? a lot of questions arising in here: decoherence effects, control inputs adjustment, and so forth.

Look forward to a well convinced experimental feedback of theoretic conclusion. Our works tendency is on controlling of BEC and physical particles occurred in lab experiment.

\section{ACKNOWLEDGMENTS}

The author thank to ACS National Meetings \& Exposition for posters

\section{REFERENCES}

[1] A. Bulatov, B. E. Vugmeister and H. A. Rabitz, Nonadiabatic control of Bose-Einstein condensation in optical traps, Physical Review A 60(6), 1998, pp 4875-4881.

[2] R. Dautray and J. Lions, Mathematical Analysis and Numerical Methods for Science and Technology, Vol. 5, Evolution Problems I, Berlin-Heidelberg-New York. Springer-Verlag, 1992.

[3] J. Grond, et al, Optimal control of trapped Bose-Einstein condensates, Physical Review A 80, 2009, p. 053625.

[4] J. L. Lions, Optimal Control of Systems Governed by Partial Differential Equations, Berlin-Heidelberg-New York, Springer-Verlag, 1971.

[5] N. Parker, N. Proukakis, C. Barenghi and C. Adams, Controlled vortex sound interactions in atomic Bose-Einstein-Condensates, Physical Review Letters 92(6), 2004, p.160403

[6] L. Pitaevskii, Bose-Einstein Condensation, Internat. Ser. Monogr. Phys. 116, Clarendon Press, Oxford UK, 2003.

[7] S. A. Rice, Optical Control of Molecular Dynamics, New York, Wiley, 2000.

[8] S. Sklarz and D. Tannor, Loading a Bose-Einstein-condensate onto an optical lattice: An application of optimal control theory to the nonlinear Schröinger equation, Physical Review A 66, 2002, p. 053619.

[9] J. Stickney, Increasing the coherence time of Bose-Einstein-condensate interferometers with optical control of dynamics, Physical Review A 75, 2007, p. 063603.

[10] R. Temam, Infinite-Dimensional Dynamical Systems in Mechanics and Physics, Second Edition, Applied Mathematica Sciences Vol. 68, Berlin-Heidelberg-New York, Springer-Verlag, 1997.

[11] S. Trotzky, Time resolved observation and control of superexchange interactions with ultracold atoms in optical lattices, Science 319, 2008, p.295.

[12] Q. F. Wang, C. Cao, Quantum numerical optimal control of nucleon and meson dissipative dynamical model, Proceeding of the European Control Conference, 2009, pp 168-172.

[13] Q. F. Wang and V. P. Belavkin, Quantum control for Bose Einstein condensates, International Scientific Conference on Physics and Control. 2009.

[14] Q. F. Wang, Bose-Einstein-Condensates and its control problems, 245th ACS National Meeting \& Exposition, Poster, 2013.

[15] Q. F. Wang, Quantum optimal control for Bose-Einstein-condensates with demonstration, Cybernetic and Physics 1(2), 2012, pp 144-153.

[16] Q. F. Wang, Optimal Control for Nonlinear Parabolic Distributed Parameter System, Lambert Academic Publishing, 2011.

[17] Q. F. Wang, Practical Application of Optimal Control Theory: computational approach, Lambert Academic Publishing, 2011. 